\title{
A Variable Turbulent Schmidt Number Formulation for Scramjet Application
}

\author{
X. Xiao, J. R. Edwards; H. A. Hassan ${ }^{\ddagger}$ \\ North Carolina State University, Raleigh, NC 27695 \\ A. D. Cutler $\S$ \\ The George Washington University, Hampton, VA 23666
}

\section{Introduction}

In high speed engines, thorough turbulent mixing of fuel and air is required to obtain high performance and high efficiency. Thus, the ability to predict turbulent mixing is crucial in obtaining accurate numerical simulation of an engine and its performance. Current state of the art in CFD simulation is to assume both turbulent Prandtl number and Schmidt numbers to be constants. However, since the mixing of fuel and air is inversely proportional to the Schmidt number, a value of 0.45 for the Schmidt number will produce twice as much diffusion as that with a value of 0.9. Because of this, current CFD tools and models have not been able to provide the needed guidance required for the efficient design of a scramjet engine.

The goal of this investigation is to develop the framework needed to calculate turbulent Prandtl and Schmidt numbers as part of the solution. This requires four additional equations: two for the temperature variance and its dissipation rate and two for the concentration variance and its dissipation rate. In the current investigation emphasis will be place on studying mixing without reactions. ${ }^{1,2}$ For such flows, variable Prandtl number does not play a major role in determining the flow. This, however, will have to be addressed when combustion is present.

The approach to be used is similar to that used to develop the $k-\zeta$ model. $^{3}$ In this approach, relevant equations are derived from the exact Navier-Stokes equations and each individual correlation is modeled. This ensures that relevant physics is incorporated into the model equations. This task has been accomplished. The final set of equations have no wall or damping functions. Moreover, they are tensorially consistent and Galilean invariant. The derivation of the model equations is rather lengthy and thus will not be incorporated into this abstract, but will be included in the final paper.

As a preliminary to formulating the proposed model, the original $k-\zeta$ model with constant turbulent Prandtl and Schmidt numbers is used to model the supersonic coaxial jet mixing experiments involving $\mathrm{He}, \mathrm{O}_{2}$ and air of Refs. 1 and 2. This step is important in order to evaluate the underlying turbulence model especially because of some discrepancies noted in comparing theory and experiment in Refs. 1 and 2. Comparisons are made with velocity, concentration, stagnation pressure and temperature at various stations in the jet. The results indicate sensitivity to Schmidt number. Moreover, the discrepancies noted in Ref. 1 and 2 are a result of the underlying $k-\omega$ model used and are absent in the current model.

\footnotetext{
*Post-Doctoral Research Associate, Member AIAA.

${ }^{\dagger}$ Associate Professor, Senior Member AIAA.

${ }^{\ddagger}$ Professor, Associate Fellow AIAA.

$\S$ Associate Professor, Senior Member AIAA.
} 


\section{Results and Discussion}

Comparison are made with the experiments of Refs. 1 and 2. In these experiments, a coaxial nozzle was designed to produce two uniform, coaxial jets at its exit. The center flow consists of $95 \% \mathrm{He}, 5 \% \mathrm{O}_{2}$ and a Mach number $M=1.8$, while the outer flow is that of air at $M=1.8$. Velocity, pitot pressure, composition, and total temperature were measured at various stations.

The grid employed is identical to that used in Refs. 1 and 2. It consists of 188,080 cells and is decomposed into 13 blocks for parallel computing. An axisymmetric finite volume solver is employed to simulate the flow, where a second order ENO(Essentially Non-Oscillating) upwinding method based on the Low Diffusion Flux Splitting scheme of Edwards ${ }^{4}$ is used to discretize the invisid fluxes while central differences are used for the viscous and diffusion terms. Planar relaxation is employed and the code is parallelized using domain decomposition and message passing(MPI) strategies.

As was done in Ref. 1 and 2, the range of $r$ in the plots is truncated to show more clearly the region of interest. In general, good agreement is indicated beyond the range shown in the figures.

Figures 1-4 compare calculations and measurements for the $\mathrm{He}-\mathrm{O}_{2}$ mass fraction, velocity, pitot pressure and temperature. Calculations were carried out for two sets of turbulent Schmidt numbers. As is seen from the figures, it appears that no single Schmidt number will fit the data thus demonstrating the need for a turbulent Schmidt number formulation. All calculations presented here assume a turbulent Prandtl number $\left(P r_{t}\right)$ of 0.89 .

Figure 1 compares computed and measured mass fractions at selected stations. As is seen from the figure, a Schmidt number of 0.9 gives better agreement with experiment. In general, calculations underpredict experiment near the axis and slightly overpredict away from the axis. Note also that there is no discontinuity in the slope of the mass fraction for any of the cases considered as was noted in Refs. 1 and 2 using the $k-\omega$ model. As is seen from the figure, calculated results are rather sensitive to the turbulent Schmidt number near the axis; a reduced value of the mass fraction is noted at the lower Schmidt number because of enhanced diffusion.

The mean velocity is shown in Fig. 2. At $x=2 \mathrm{~mm}$, the velocity profile is a result of merging of the coflow nozzle inner surface boundary layer with the region of separation at the lip and the shock wave emanating from the lip. Downstream, the calculated velocity is in good agreement with the measurements and is not sensitive to the Schmidt number.

The pitot pressure is shown in Fig. 3. As is seen from $x=3 \mathrm{~mm}$ station, there is a layer with reduced pitot pressure at the boundary between the center jet and the coflow. Downstream, the center jet spreads with the pressure near the axis falling and then rising in the wake of the nozzle lip. The figure shows that the pitot pressure is somewhat sensitive to the Schmidt number near the axis. This behavior is a result of the behavior of the $\mathrm{He}-\mathrm{O}_{2}$ mass fraction indicated in Fig. 1.

The measured and calculated total temperature is shown in Fig. 4. Measurements ${ }^{1,2}$ indicate that the gas supply temperature varied substantially from run to run. Because of this, it was recommended that calculations employ the experimentally measured temperature for that run and not the average temperature over many runs as was done for other parameters. When the probe errors ( of the order of 1\%) are taken into consideration, it is seen that agreement with experiment is acceptable. However, results are Schmidt number dependent.

\section{Concluding Remarks}

The above results demonstrate that the underlying turbulence model does not exhibit the shortcomings of the $k-\omega$ model and indicate the need for a variable Schmidt number formulation. This will be the subject of the final paper. As indicated earlier, all relevant equations and modeling is complete and we are in the process of determining model constants. 


\section{Acknowledgment}

The authors from North Carolina State University would like to acknowledge support under NASA grant NAG-1-03030.

\section{References}

${ }^{1}$ Cutler, A. D., Carty, A. A., Doerner, S. E., Diskin, G. S., and Drummond, J. P., "Fundamental Mixing and Combustion Experiments for Propelled Hypersonic Flight," AIAA Paper AIAA-2002-3879, July 2002.

${ }^{2}$ Cutler, A. D., Diskin, G. S., Danehy, P. M., and Drummond, J. P., "Supersonic Coaxial Jet Experiment for CFD Code Validation," AIAA Paper AIAA-99-3588, July 1999.

${ }^{3}$ Robinson, D. F. and Hassan, H. A., "Further Development of the $k-\zeta$ (Enstrophy) Turbulence Closure Model," AIAA Journal, Vol. 36, No. 10, 1998, pp. 1825-1833.

${ }^{4}$ Edwards, J. R., "A Low Diffusion Flux Splitting Scheme for Navier-Stokes Calculations," Computers 83 Fluids, Vol. 26, No. 6, 1997, pp. 635-659. 


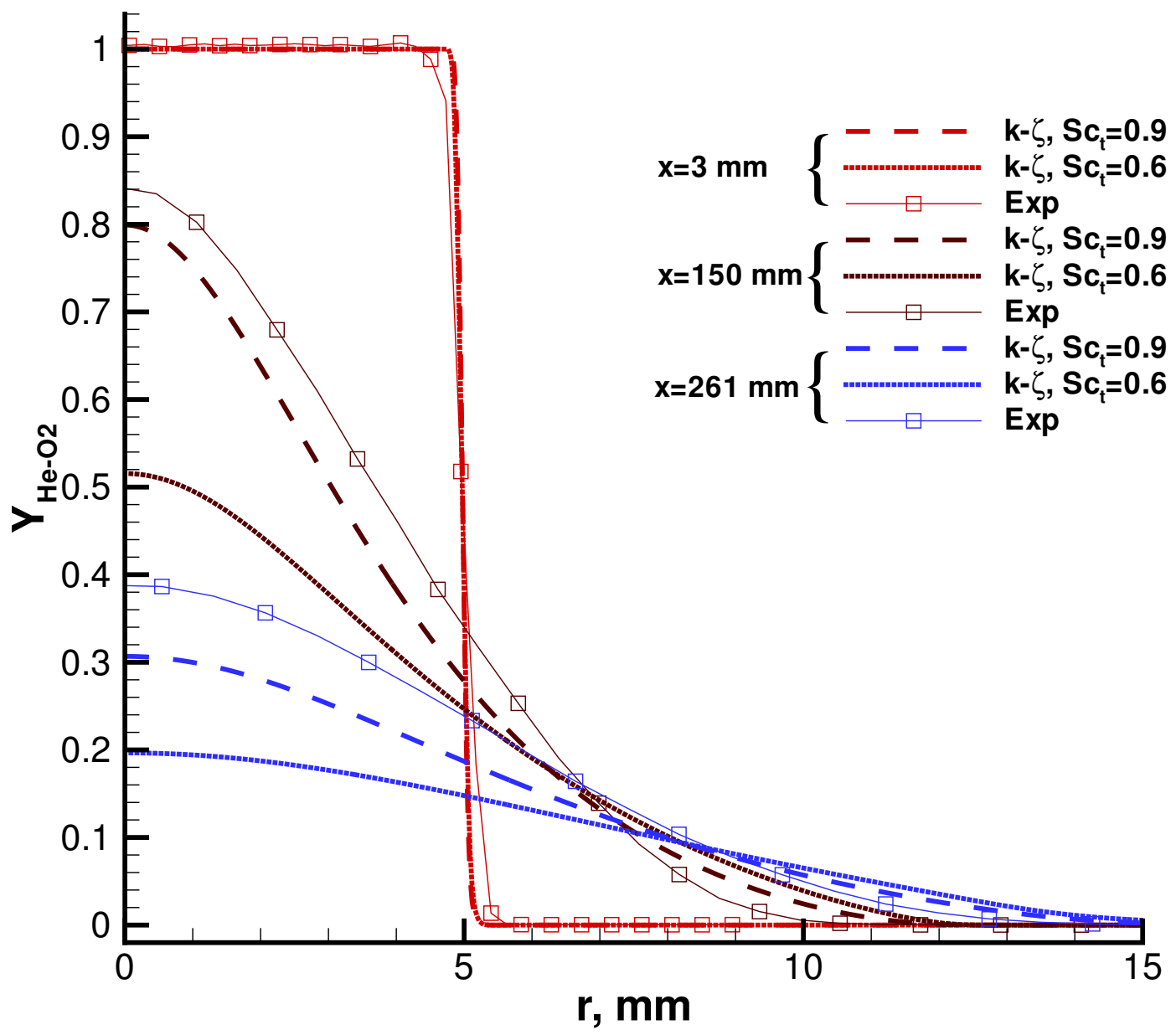

Figure 1. Comparison of mass fraction with experiment 


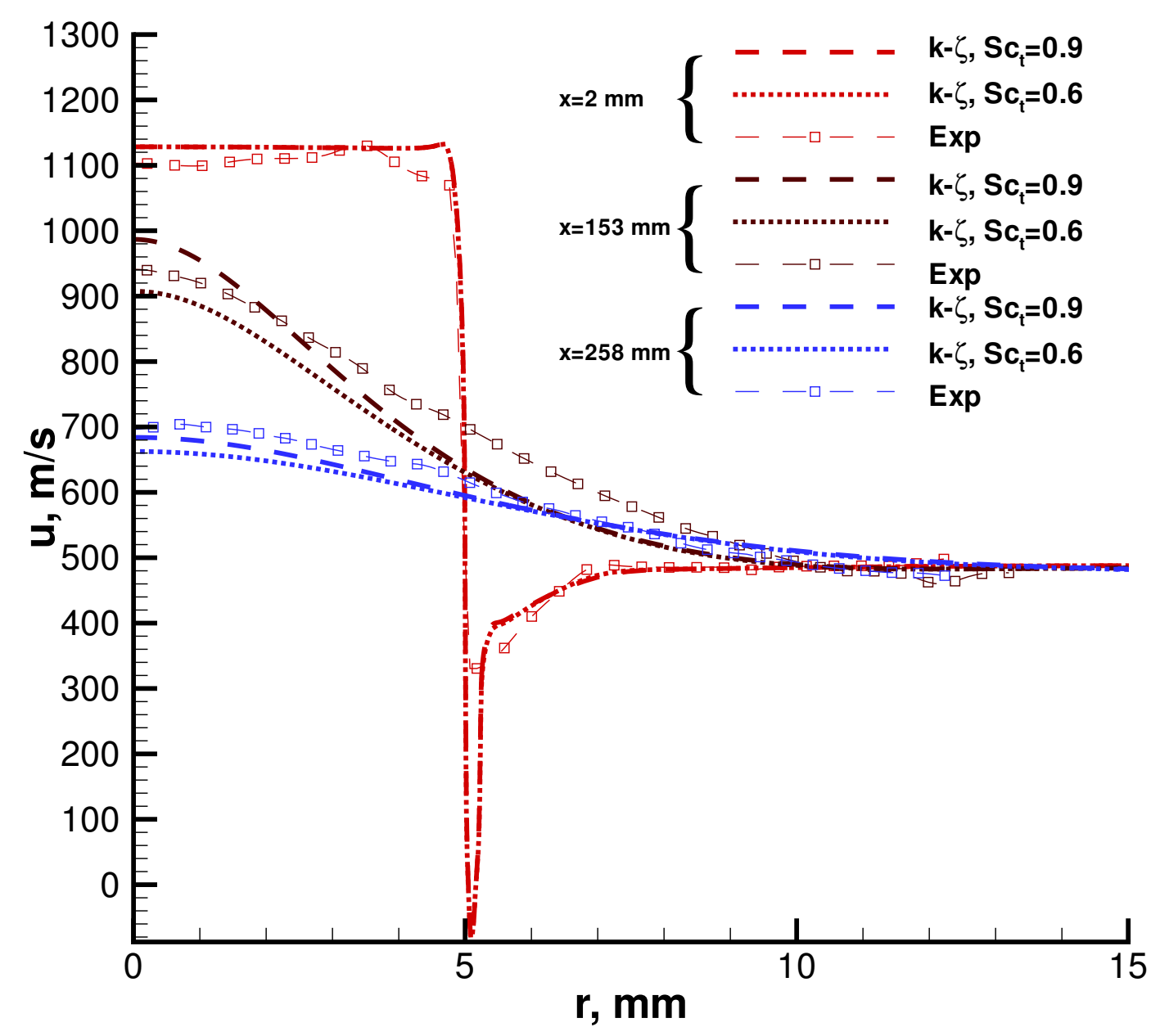

Figure 2. Comparison of mean axial velocity with experiment 


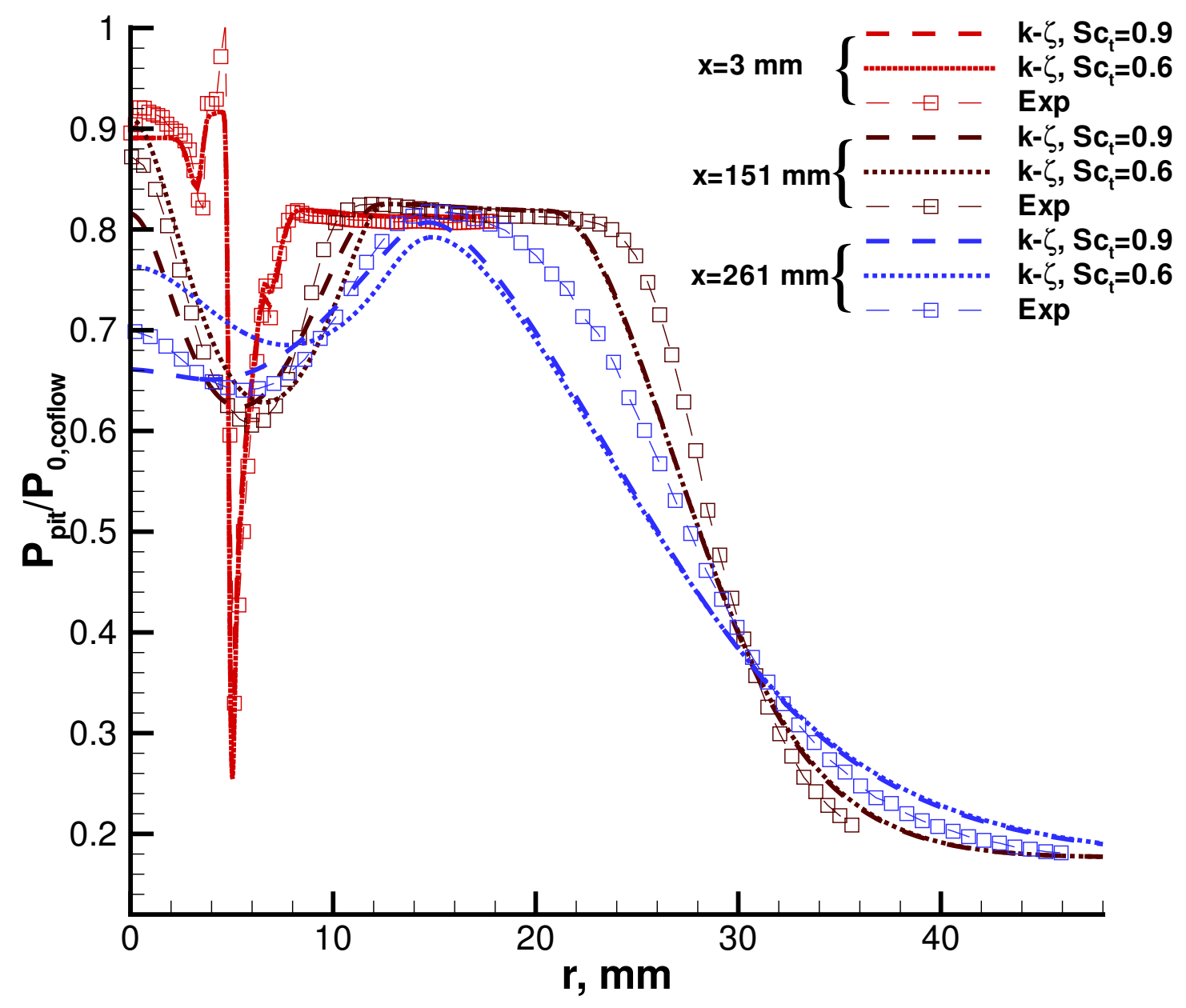

Figure 3. Comparison of pitot pressure with experiment 


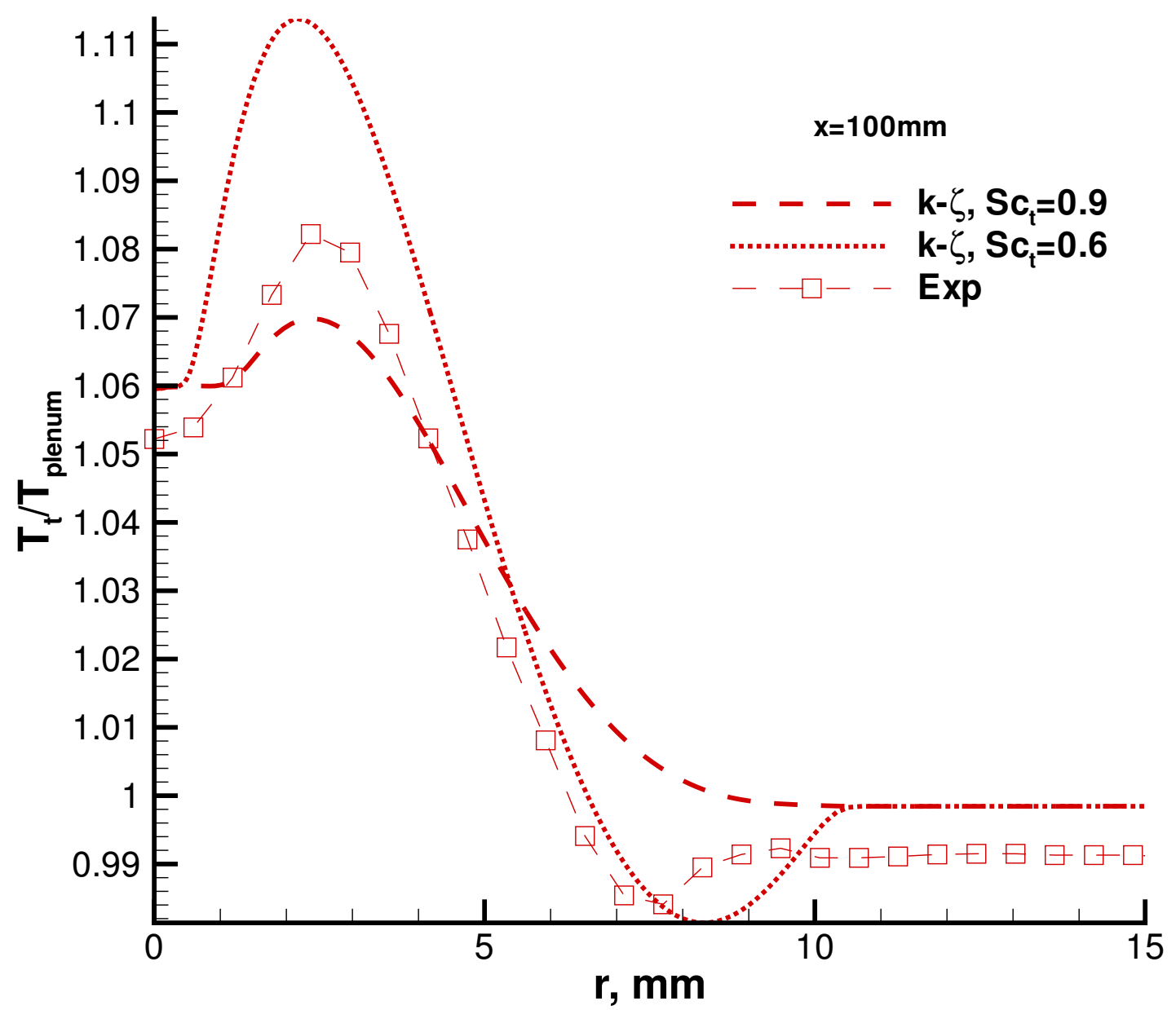

Figure 4. Comparison of total temperature at $\mathrm{x}=100 \mathrm{~mm}$ with measurements 